\title{
Téoros
}

Revue de recherche en tourisme

\section{La nordicité}

\section{Un défi, un atout, une expérience}

\section{Annie Béliveau}

Volume 18, numéro 2, été 1999

La nordicité

URI : https://id.erudit.org/iderudit/1072102ar

DOI : https://doi.org/10.7202/1072102ar

Aller au sommaire du numéro

Éditeur(s)

Université du Québec à Montréal

ISSN

0712-8657 (imprimé)

1923-2705 (numérique)

Découvrir la revue

\section{Citer cet article}

Béliveau, A. (1999). La nordicité : un défi, un atout, une expérience. Téoros, 18(2), 37-40. https://doi.org/10.7202/1072102ar

Ce document est protégé par la loi sur le droit d'auteur. L'utilisation des services d'Érudit (y compris la reproduction) est assujettie à sa politique d'utilisation que vous pouvez consulter en ligne.

https://apropos.erudit.org/fr/usagers/politique-dutilisation/
Cet article est diffusé et préservé par Érudit.

Érudit est un consortium interuniversitaire sans but lucratif composé de l'Université de Montréal, l'Université Laval et l'Université du Québec à Montréal. Il a pour mission la promotion et la valorisation de la recherche. https://www.erudit.org/fr/ 


\section{LA NORDICITÉ}

\section{UN DÉFI, UN ATOUT, UNE EXPÉRIENCE}

\section{Annie Béliveau}

66"33' $07^{\circ}$ "Nord, $35^{\circ} 50^{\prime} 51^{\prime \prime}$ Est. Décembre. Température moyenne : environ $=15^{\circ} \mathrm{C}$. Napapiiri : le cercle polaire. Au solstice d'hiver, le soleil ne franchit plus la ligne d'horizon. Il se lève bien quelque part plus au sud, mais ici seule une fable lueur perce l'horizon pour quelques heures. La nature giveé, la blancheur Éclatante de la neige semblent davantage unles à guider les pas des promeneurs. Puis lé ciel s'enveloppe d'un manteau feutré, passant langoureusement du bleu velouté à la noirceur. C'est ce que l'on appelle en finnois kaamos ou * le moment bleu *. Puis, certains soirs, la nuit dejà parseméé d'étoiles s'illumine de tracés verts, bleus, violacés... ce sont les aurores boréales porteuses de légendes, de mythes pour certaines cultures, de fascination pour les autres.

Le mot Laponie captive à lui seul l'imagination, suggérant un environnement étrange auquel appartiennem les vastes tendues de la Laponie finlandaise, profondément associées au peuple et à la culture sâmes. Cette immense contrée sawnage, avec ses monts arrondis, ses marais et ses prairies qui se déroulent à l'infini, ses lacs silencieux et ses larges rivières étincelantes, exerce un attrait grandissant sur les citadins européens et accueille des touristes de plus de plus nombreux'.

C"est ainsi que Le Guide de Tourisme Michelin présente la Laponie finlandaise. Cette région s'étend sur quelque 100000 $\mathrm{km}^{2}$. Le cercle polaire traverse le premier quart de cette vaste étendue, passant de la forct mixte à la forêt de conifere, puis a la toundra... Quelque 200000 personnes $^{2}$ y vivent, dont environ 4000 Sâmes (les autochtones des pays nordiques) dans le nord de la Finlande. Et chaque année des milliers de touristes de tous les coins du globe, particulièrement des pays Scandinaves, de Russie et d'Europe centrale s'y amenent.

\section{LES ATOUTS}

La Laponie dispose en fait d'une forte image presque mythique dans l'imaginaire populaire. Sa nordicité tout comme son nom semblent évoquer la pureté d'un espace sauvage, un peuple autochtone vivant encore de valeurs ancestrales, mais inévitablement aussi l'hiver, la neige, les rennes... le Père Noél. Et quoi de mieux qu'une image forte, au parfum d'authenticitế, pour éveiller l'intếrêt de voyageurs aguerris ? Cette même combinaison assor- tie d'une stratégie et d'un programme de promotion concertée...

Une telle association d'idếes, les \& têtes pensantes $x$ du tourisme finlandais l'ont mise à profit dès le début des années 1980. En effet, les activités et les attraits de la Laponie étant jugés insuffisants pour soutenir le développement touristique de la région, il fallait trouver une image porteuse ${ }^{3}$. L'idée d'en faire a le pays du Père Noẻl $* s^{\prime}$ 'est ainsi implantée, puis fut mise en marché dans le monde entier. Partout on sème le message que LE Père Noèl, le vrai, vient de Finlande. En Laponie, il a son village complet : son bureau de poste, son atelier et son bureau où l'on peut le rencontrer, ses (nombreuses !) boutiques de cadeaux et, maintenant, son pare d'amusement, Sania Park.

Même les transporteurs s'y mettent : l'aéroport international de Rovaniemi arbore de magnifiques sculptures dorées de rennes volants, Finnair est la ligne aérienne officielle du Père Noël (on peut mème voir son image sur certains avions de la com-



Les sculptures représentant les rennes du Père Noel, Aéroport International de Rovamieni 
TABLEAU 1

L'évolution des entrées touristiques en Finlande

\begin{tabular}{|c|c|c|c|c|c|}
\hline Rtgion/Viut & $\begin{array}{c}\text { JanviEa } \\
\text { DécEMese } 1996\end{array}$ & $\begin{array}{c}\text { Janvier } \\
\text { Décemske1 } 1997\end{array}$ & $\begin{array}{l}\text { \% vaRiation } \\
1996 \text { vs. } 1997\end{array}$ & $\begin{array}{l}\text { \% HWER SLIA AN } \\
\text { (NOV. } 96 \text { A MARS 97) }\end{array}$ & $\begin{array}{l}\text { \%) ET SUR AN } \\
\text { (JUIN A ADUT 97) }\end{array}$ \\
\hline Toute la Finlande & 3284644 & 3645854 & $11,00 \%$ & $27 \%$ & $46 \%$ \\
\hline - Helsinki & 1229381 & 1350110 & $9,82 \%$ & $29 \%$ & $38 \%$ \\
\hline - Jaensua (Carélie du Nard) & 15929 & 18463 & $15,91 \%$ & $23 \%$ & $41 \%$ \\
\hline - Région de Kussama & 24920 & 22333 & $-10,38 \%$ & $38 \%$ & $51 \%$ \\
\hline Laponie & 401234 & 399907 & $-0,33 \%$ & $44 \%$ & $43 \%$ \\
\hline - Rovaniemi & 139151 & 133924 & $-3,76 \%$ & $50 \%$ & $37 \%$ \\
\hline - Pyhä - Luosto & 11703 & 9108 & $-22,17 \%$ & $62 \%$ & $31 \%$ \\
\hline - Kemi & 11370 & 10321 & $-9,23 \%$ & $49 \%$ & $32 \%$ \\
\hline - Kittilä (Levi) & 34499 & 31760 & $7,94 \%$ & $64 \%$ & $22 \%$ \\
\hline - Kolari (Yilias) & 16321 & 18995 & $16,38 \%$ & $51 \%$ & $41 \%$ \\
\hline - Muonio (Pallas) & 27488 & 30912 & $12,46 \%$ & $59 \%$ & $19 \%$ \\
\hline - Saariselkä & 62247 & 71877 & $15,47 \%$ & $42 \%$ & $44 \%$ \\
\hline
\end{tabular}

Source: Office du tourisme de la Finlande (M.E.K.)

pagnie), et les trains à destination de Rovaniemi se nomment * Santa Claus Express w. Ajoutons les nombreux efforts promotionnels conjoints des quelque 23 compagnies qui coopérent au Triangle de Noël et l'on se trouve avec une recette gagnante en terme d'accroissement de l'achalandage touristique.

A Rovaniemi, les entrées touristiques pour I'hiver 1996-97 représentaient $50 \%$ de l'achalandage annuel comparativement à $44 \%$ pour la Laponie et $27 \%$ pour l'ensemble de la Finlande. Cette année, avec l'ouverture de Santa Park, on a vécu une nette augmentation, notamment à cause de la centaine de vols nolisés spécialement pour son ouverture officielle, mais également pour l'ensemble de la saison de Noël. Décembre 1998 amenait donc un total de quelque 35000 touristes, pour la plupart britanniques, mais également des Russes, des Italiens et des Français ${ }^{4}$.

Construit à l'intérieur d'une grotte à l'entrée de la forèt, Santa Park veut recréer toute l'année l'ambiance de Noël. Au cour du site, surmontant le Carrousel du Père Noèl, les visiteurs voient un tableau électronique qui affiche le décompte des jours avant le prochain Noël. Les lutins accueillent les visiteurs et les invitent à s'amuser grâce aux quelques manèges ou aux jeux d'adresse, au spectacle multimédia ou encore au théâtre de marionnettes racontant tous deux une histoire de Noẻl. Tous les éléments étant à l'intérieur de la grotte, on minimise les effets sur l'environnement en plus d'offrir un site * à l'épreuve des intempéries... et un joyau pour l'anchitecture finlandaise $3^{5}$.
Par contre, malgré la force d'attraction du cercle polaire et du Pêre Noël, les nombreux séjours à Rovaniemi sont généralement très courts. La majorité des Britanniques, puisqu'il s'agit de la plus importante clientèle en décembre, reste au plus 24 heures et le plus souvent en fait, moins de 10 heures. Beaucoup font en effet une excursion en Concorde pour rencontrer Father Christmas... pour la modique somme d'environ $4000 \$$ par adulte ${ }^{6}$ ! Outre leur rencontre avec l'être mythique, ces petites familles anglaises font l'expérience de la motoneige, du traîneau à chiens, du traîneau à rennes, puis d'un banquet de Noël avant leur vol de retour.

\section{LE DEFI}

Pour tenter de prolonger le séjour des touristes, la coopération promotionnelle doit s'étendre au-delà de la région limitrophe au cercle polaire. On vend donc egalement la possibilité de faire du ski alpin aux montagnes de Levi, d'Ylläs, de Pallas et de Saariselkä ${ }^{7}$. Les trois premières sont les plus hautes montagnes de la Finlande (altitude moyenne de $370 \mathrm{me}$ tres), avec des pistes quil s'étendent jusqu'à 2400 et 3000 mètres. Saariselkä est la plus modeste avec ses 180 mètres d'altitude, mais il s'agit d'un des plus anciens centres touristiques de la région. Ceci explique en partie que cette destination traditionnelle se trouve au deuxième rang des nuitées touristiques en Laponie (voir tableau 2), suivi du secteur de Levi, la montagne « à la mode .

Dans tous les centres de villégiature de la Laponie, des banquets traditionnels sont organisés la veille de Noël. Et, bien sûr, la soirée ne se termine pas sans la visite de l'invité d'honneur de cette nuit magique, souvent accompagné d'un renne... La veille du Nouvel An, on invite plutôt les touristes à découvrír un peu la culture lapone. On organise alors, avant minuit, une sortie vers une kota, tente lapone, où un chaman lira l'avenir dans l'étain fondu.

Dans ces centres de villégiature, le faible achalandage (en raison de l'isolement et

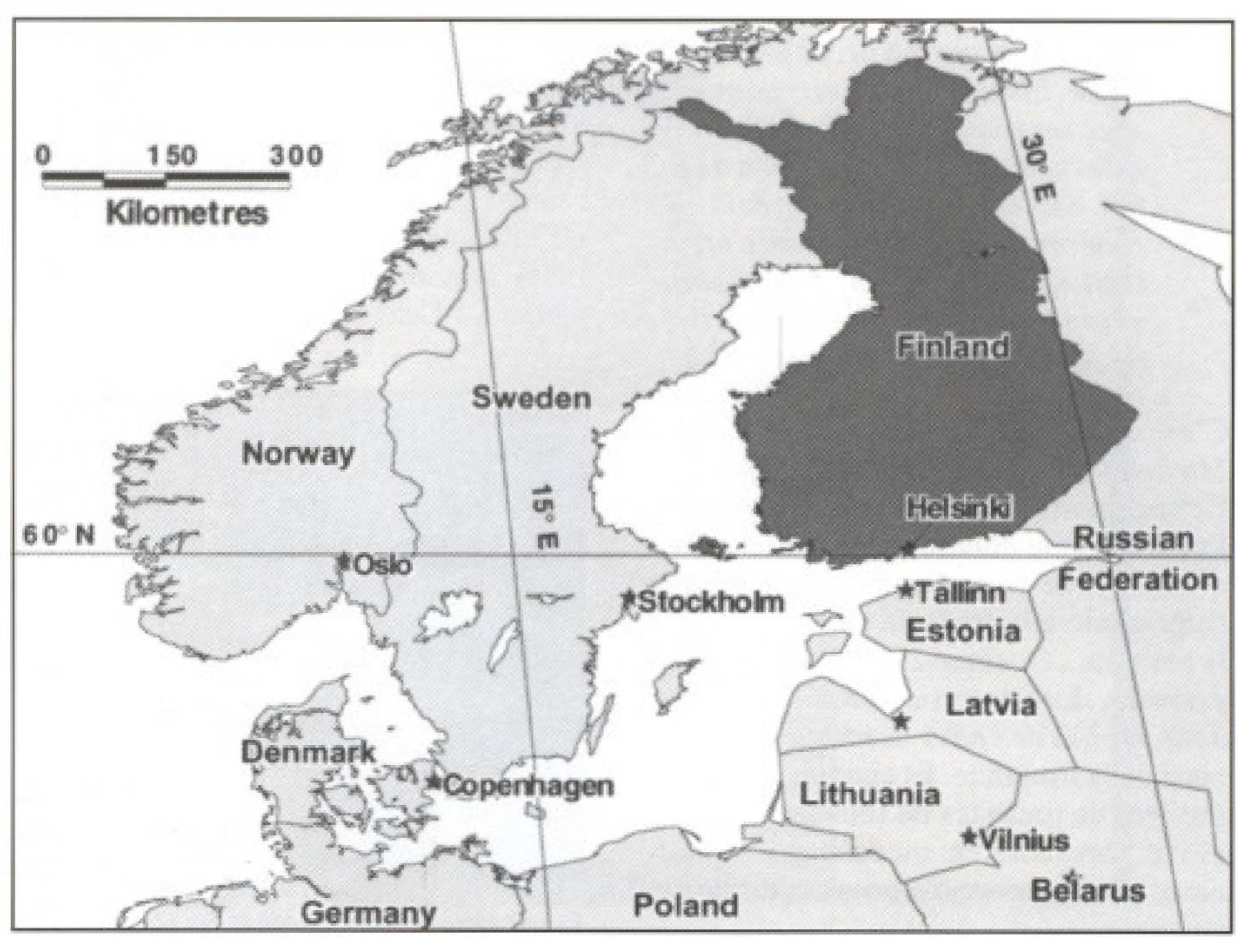


TABLEAU 2

Nuitées dans certaines régions finlandaises pour les marchés d'importance

Décembre 1997 - mars 1998

\begin{tabular}{|c|c|c|c|c|c|c|c|c|c|}
\hline Reanow & Toutes phovenances & FinLande & Autaes phovenasicts & Russif & Bopaume Umi, lalamoe & Aluemagane & Japan & Frasce & IraLie \\
\hline Toutes régions & 1684730 & 1187081 & 497649 & 181814 & 42949 & 37730 & 17689 & 16262 & 11534 \\
\hline Sud de La Finlande & 514022 & 279644 & 234378 & $\$ 5296$ & 17278 & 12480 & 5534 & 5593 & 5075 \\
\hline - Helsinki & 306597 & 129030 & 177581 & 61490 & 13778 & 7726 & 5005 & 4092 & 4231 \\
\hline Carelie do Nond & 41679 & 33306 & 8373 & 4291 & 216 & 935 & 140 & 155 & 118 \\
\hline - JDensuu & 15224 & 11223 & 4001 & 1881 & 128 & $37 \gamma$ & 137 & 109 & 118 \\
\hline Laponie & 201595 & 103809 & 97786 & 18942 & 18019 & 12157 & 9456 & 8152 & 3466 \\
\hline - Rovaniemi & 57362 & 19168 & 38194 & 10111 & 3255 & 2772 & 3121 & 2989 & 2504 \\
\hline - Prhä-Luosto & 12759 & 8043 & 4716 & 257 & 3188 & 214 & 124 & 211 & 20 \\
\hline - Keni & 9649 & 7529 & 2119 & 108 & 56 & 60 & 20 & 234 & 203 \\
\hline - Kittilla (Levil & 32923 & 18259 & 14664 & 2711 & 6454 & 869 & 2679 & 59 & 5 \\
\hline - Kolari |YIIăs| & 15295 & 11550 & 3745 & 502 & 1525 & 302 & 934 & 17 & 0 \\
\hline - Sagriselka & 39596 & 23052 & 16544 & 3190 & 1674 & 3813 & 2185 & 828 & 390 \\
\hline
\end{tabular}

Source : Office du tourisme de la Finlande (M.E.K.)

de la faible notoriété individuelle) est en quelque sorte compensé par des séjours plus longs que dans les quelques villes nordiques comme Rovaniemi. On s'y paie des séjours de détente, des séjours de ski de randonnée, des séjours multi-activites. mais surtout, la cabane dans le Nord.

Surmontant les défis nordiques, l'éloignement, les rigueurs de l'hiver et les faibles reliefs, cette région obtient un meilleur taux d'achalandage hivernal que bien des destinations nordiques... dont le Québec. Bien que le volume annuel de touristes étrangers soit comparable, tout comme la majorité des activités hivernales offertes, force est de constater que la Finlande et surtout la Laponie, accueillent une plus forte proportion de visiteurs durant l'hiver que le Québec (comparer les tableaux 1 et 3) et ce, peu importe le marché d'origine étudié.

\section{L'EXPÉRIENCE}

Partie étudier les similitudes et les différences entre le tourisme hivernal en Finlande et au Québec, j"ai pu vivre l'expérience de la Laponie à deux reprises. Contrairement à ce que l'on connaît ici, le Grand Nord finlandais est des plus accessibles et ce, à une gamme de prix pouvant accommoder toutes les bourses. Que ce soit par avion, par train ou par autobus, des connections sont possibles tous les jours, été comme hiver.

Le premier voyage m'a permis de vivre, en tant que touriste, l'expérience d'un séjour de détente autour de la période de Noël : un forfait vendu par Lapland TraveP incluant le transport, l'hébergement et les petits déjeuners scandinaves pour quatre jours pour environ $500 \mathrm{~S}^{10}$.

D'abord un train de nuit nous amène de Helsinki à Rovaniemi puis, au petit matin, vers Kemijärvi ; ensuite, un autobus nous conduit à la porte de Sallatunturin Tuvat. Ce centre de villégiature, en marge de la petite municipalité de Salla, est situé près de la frontière russe, à une trentaine de kilomètres au-dessus du cercle polaire. Nous sommes hébergés dans un un chalet tout équipé... avec sauna bien sûr ${ }^{11}$. Le ski de fond (accès gratuit) est à la porte du mökki ${ }^{12}$, alors que la petite montagne se trouve à quelques minutes de marche pour le ski alpin ou encore une initiation au telemark.
Sur place, Arctic Circle Safaris offre des safaris motoneige et des excursions à la ferme de rennes. On peut apprendre à conduire un traîneau à chiens, un traîneau attelé à un renne, ou encore simplement à explorer les sentiers du site en ski de fond ou en raquettes. Ces ballades libres peuvent d'ailleurs donner lieu à d'intéressantes rencontres : les rennes, en Laponie, constituent une espèce semi-sauvage. On en fait l'élevage, mais ils vagabondent comme bon leur semble sur le territoire... leur territoire !

Outre cette espece singulièrement peu farouche, un autre fait contribue à rendre cet environnement naturel des plus singuliers et apaisants : l'ensoleillement... ou plutôt son absence. En effet, en ce temps de l'année, les journées sont les plus courtes. Le soleil ne franchit pas la ligne d'ho-

\begin{tabular}{|c|c|c|c|c|c|c|c|}
\hline & & & TABL & AU 3 & & & \\
\hline Volur & ne touris & que au 0 . & vec pour & es grands & archés int & rnationas & \\
\hline & & ONNÉES RÉ: & ES (STATIS & IOUE CANAD & & ESTIMATI & POUR 1997 \\
\hline Mariches d'onionase & 1995 & 1996 & 1997 & $\begin{array}{l}\text { Nuntes PRA } \\
\text { Worat } 1995\end{array}$ & 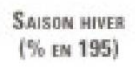 & $\begin{array}{l}\text { EwTiEts } \\
\text { HIVER }\end{array}$ & $\begin{array}{l}\text { Numites } \\
\text { Aiver }\end{array}$ \\
\hline Etats-Unis & 1946400 & 1920000 & 1868000 & 3,4 & $23,1 \%$ & 431508 & $1467 \quad 127$ \\
\hline France & 366200 & 390000 & 384000 & 10,1 & $16,8 \%$ & 64512 & 651571 \\
\hline Rovaume-Uni & 113600 & 118000 & 125000 & 4.6 & $16,8 \%$ & 21000 & 96600 \\
\hline Allemagne & 78100 & 107000 & 86000 & 6,1 & $16,8 \%$ & 14448 & 83 133 \\
\hline Total infernational & 3041000 & 3162000 & 3119000 & $\therefore$ & $18,4 \%$ & 573116 & - \\
\hline
\end{tabular}

Sources : Statistiques Canada al estimations baseses sur les entrees de 1997 et les proportions observees pour I'hiver 1995

Calculer une moyenne avec les mpyannes de nuitées des touristes des différentes provenances présentées n'aurait aucune valeur vu leur divarsite. 
rizon. Pendant à peine deux ou trois heures c'est la pénombre; le reste du temps, il fait nuit. Une partie des sentiers de ski de fond est done éclairée... jusqu'à plus de 10 heures par jour!

Comme il se doit, la veille de Noël, un banquet attend tous les invités de Sallatunturin Tuvat soit, cette fois, des Finlandais, un Français, deux Québécois et un groupe de Sud-Africains. Divers plats à base de viande de rennes sont évidemment au menu, ainsi que des plats de saumon. Un typique souper-buffet de Noël couronné, bien sûr, par la visite du Père Noël accompagné d'un renne et d'un lutin !

Outre les ressources mises en valeur ( $l a$ nature, le Père Noèl...), ce séjour a permis de constater le souci accordé a l'intégration des infrastructures au paysage : les chalets de bois du centre de villégiature étaient tous peints d'un gris-bleu de telle sorte qu'ils se mariaient avec le ciel d'hiver ; l'épicerie, située entre la petite route et la montagne de ski, était aménagée à l'intérieur $\mathrm{d}^{+}$un chalet de bois rond; l'hôtel Revontuli, au pied de la montagne, représentait les aurores boréales (il s'agit d'ailleurs de la signification de son nom). La structure blanche et basse de son bâtiment principal, bien que moderne, semblait se fondre avec la montagne, en arrière plan.

L'un des principes finlandais en matière d'aménagement touristique veut, en effet, que les hôtels (enire autres) ne soient pas des structures trop visibles. Lorsque situés en haut d'une montagne par exemple, ceux-ci ne doivent pas être visibles d'en bas, ni de la ville. Ceci semble done imposer des structures basses dont les couleurs s'harmonisent avec le paysage.

C'est lors du second séfour en Laponie que j'ai appris ce point de la réglementation. Ce sejour visait à rencontrer des intervenants touristiques dans le triangle de Noel, autour de Rovaniemi et du cercle polaire. Logée à Rovaniemi même, j'ai pu appré̉cier les sentiers piétonniers traversant la ville et les indications vers les points d'intérêt, mais, surtout, le phénomène kaamos.

J'ai rencontrés les dirigeants de compagnies de safaris, d'attraits touristiques axés sur le Père Noël, d'attraits culturels et des spécialistes du tourisme, notamment pour ce secteur géographique. Ces entrevues ont permis de cerner l'importance du tourisme hivernal à Rovaniemi, mais également l'importance et l'efficacité apparente de la stratégié basée sur le Père Noël.

\section{CONCLUSION}

Des lacunes subsistent toutefois quant à la répartition de la clientèle dans la saison et sur tout le territoire. Le triangle de Noël ne représente en effet qu'une infime portion du territoire lapon. Les forfaits offerts par les grossistes étrangers dans le reste de la région finissent donc par ressembler à ceux offerts sur les autres destinations nordiques, particulierement celles qui ne peuvent appuyer leur offre sur des montagnes époustouflantes. Toutefois, l'achalandage marqué d'un site éloigné, tel que Saariselkd, laisse croire qu'une distinction, un atout subsiste... l'image mythique du Grand Nord.

Accessible, tout en offrant une nature encore aussi intacte que souhaitée, la nordicité devient un atout. Jumelée à des stratégies de mise en marché et de communication cohérentes, elle est alors capable d'attirer les touristes qui désirent vivre une expérience hors du commun: l'expérience du Nord.

Amie Beliveau est finissante à la Maitrise en gestion et planification du tourisme a I'UOAM et conseillère chez Guilbault et associês, Conseil, Recherche et Stratégie marketing.

\section{NOTES}

1 (1997), Guide de Tourisme Michelin : Danemark, Nonège. Suede. Finlande, Paris, $1^{\text {the }}$ édition.

2 Population de la Laponie en 1998 . 196647 personnes. Données de Tilastokeskus (Statistiques Finlande).

3 Pretes, Michael (1995), « Postmodern Tourism : The Santa Claus Industry *, Annals of Tourism Research, 22, 1, p. 1-15.

4 Données tirćes d'une entrevue avec la directrice marketing de Santa Park.

5 Citation de la directrice du marketing de Santa Park.

6 Pour ceux qui veulent épargner, il est possible de faire seulement l'un des deux vols en supersonique. Le prix s'en trouve alors diminué de près de la moitié.
7 Ces centres de ski bénéficient tous d'un aéroport en moyenne à quelque $50 \mathrm{~km}$. Levi et Ylläs sont situés à $30 \mathrm{~km}$ chacun de la ville de Kittilä, alors que Pallas est près de Muonio (60 km de Kittilia). Saariselkã est près de la ville đu même nom et est situé à quelque $30 \mathrm{~km}$ au sud de l'aéroport de Ivalo.

8 L'auteure désire à cet effet remercier Tourisme Québec, le Département d'études urbaines et touristiques de l'UQAM, les intervenants touristiques finlandais, Guilbault et associés, ainsi que son entourage pour leur support à cet ambitieux projet.

9 Agence réceptive chargée de vendre la Laponie à divers marchés, dont le marché intérieur.

10 Prix par personne, en occupation double, incluant les places dans une cabine avec couchettes à bord du train.

11 Il faut en effet savoir que le sauna fait intégralement partie de la culture finlandaise. Si un sauna privé n'est pas compris dans l'hébergement des invités, ceux-ci ont inévitablement accès à un sauna epublic a aménage sur le site.

12 Signifie chalet. en finnois. 